\title{
OPTICAL MEASUREMENTS OF GROUND-STATE PROPERTIES OF SHORT-LIVED NUCLEI IN RESONANCE CELLS
}

\author{
H.-J. KLUGE \\ Institut für Physik, Universität Mainz, D-6500 Mainz, FRG \\ and CERN, CH-1211 Geneve 23, Switzerland
}

\begin{abstract}
Hyperfine structure and isotope shift of radioactive $\mathrm{Hg}, \mathrm{Au}$ and $\mathrm{Cd}$ isotopes have been determined by optical spectroscopy. In all cases the atoms were confined in a resonance cell. The various variants of the cell technique and alternative methods as the resonance ionization spectroscopy related to the investigation of short-lived nuclei are described, and some results are discussed.
\end{abstract}

\section{Introduction}

This contribution concentrates on the work done by the Mainz group on radioactive isotopes of $\mathrm{Hg}, \mathrm{Au}$ and $\mathrm{Cd}$ confined in a resonance cell. These experiments, which started in the early seventies, allowed for the first time the investigation of isotope shifts (IS) of nuclei with half-lives in the second or minute range.

The first round of experiments made use of the RADOP technique (nuclear Radiation Detected Optical Pumping) combined with Zeeman scanning of a spectral lamp [1]. This method, which yielded the unexpected nuclear shape transition in the light $\mathrm{Hg}$ isotopes [2], was developed and applied by J. Bonn, G. Huber, H.-J. Kluge and E.-W. Otten.

The second round became possible with the development of tunable dye lasers and led back to purely optical techniques for the investigation of the even-even isotopes and the isomers in the $\mathrm{Hg}$ chain. These experiments [3] were performed by P. Dabkiewicz, H. Fischer, H.-J. Kluge, H. Kremmling, T. Kühl, E.-W. Otten and H.A. Schuessler from the Texas A\&M University, who spent some time with us. Later on, similar investigations were carried out by F. Buchinger, P. Dabkiewicz, H.-J. Kluge, A. Müller and E.-W. Otten on Cd isotopes [4]. All these experiments took advantage of the high yields offered by the on-line mass separator ISOLDE at CERN, Geneva [5]. At present we are working on the extension of these systematic measurements to the less volatile elements $\mathrm{Au}$ and Pt. A first result, obtained by H.-J. Kluge, H. Kremmling, H.A. Schuessler, J. Streib and K. Wallmeroth [6], is the isotope shift between ${ }^{195} \mathrm{Au}$ and ${ }^{197} \mathrm{Au}$, obtained by fluorescence and polarization spectroscopy at the laboratory in Mainz. Recently, the isotope shifts of ${ }^{190} \mathrm{Au}-{ }^{193} \mathrm{Au}$ 
could be measured at ISOLDE. These off-line experiments [7] were performed by G. Bollen, H.J. Kluge. H. Kremmling, R.B. Moore, H. Schaaf, J. Streib and K. Wallmeroth, where the Au atoms were confined in a high-temperature resonance cell [8] and the resonance light following laser excitation was observed. In order to achieve higher sensitivity and selectivity, the resonance ionization spectroscopy in combination with time of flight was tested by U. Krönert, H.-J. Kluge, P. Peuser, W. Ruster, N. Trautmann and $\mathrm{K}$. Wallmeroth for the elements Xe and $\mathrm{U}$ and successfully applied to a trace-analysis experiment of $\mathrm{Pu}[9]$.

Some parts of this review on laser spectroscopy of radioactive isotopes in resonance cells are identical to that given at Oak Ridge $1 \frac{1 / 2}{2}$ years ago [10], where the first conference dedicated entirely to the application of lasers to nuclear physics took place. Since then, the neutron-deficient Au isotopes have been measured and several resonance ionization experiments have been performed. The results of these experiments will be discussed with respect to the determination of nuclear ground-state properties.

\section{Advantages and drawbacks of the cell technique}

In order to investigate the hyperfine structure (HFS) and IS of unstable nuclei by optical means, it seems to be straightforward to collect a number of atoms of the isotope under investigation, store them in a resonance vessel, excite them by photons of the appropriate wave length, and observe the resulting resonance light. Indeed, this procedure has the following advantages:

- The atoms can be accumulated and easily confined until the nuclear decay takes place. During the nuclear lifetime, ideally each atom can be excited optically up to about $10^{7}$ times/s.

- The nuclear decay can easily be observed. Its angular dependence on the nuclear orientation achieved by optical pumping can be used as a monitor for the optical resonance.

- Those nuclei which are obtained as daughter products of mother atoms confined in the cell are investigated.

- Pulsed lasers with low-duty cycle can be used.

On the other hand, the cell technique has some drawbacks which have to be considered carefully:

The atoms will collide with the walls of the resonance vessel, stick there for a certain length of time, or even chemically react with the wall material and are lost for ever.

- The random walk of the atoms in the resonance vessel leads to a Doppler broadening of the resonance signal.

- The walls of the resonance cells are sources of a high background of backscattered light. 
It is difficult to give a general rule whether or not the cell storage is superior to the atomic beam methods or ion trap techniques. The most stringent limitation is clearly given by the chemical reaction with the wall material. For example, the few alkali atoms available for an isotope far from stability are completely adsorbed when hitting a wall [11]. As a consequence, they are in the vapour phase for only about $10^{-4} \mathrm{~s}$ in vacuum and about $1 \mathrm{~s}$ using 100 Torr of $\mathrm{He}$ as a buffer gas. For a limited number of $\mathrm{Au}$ atoms $\left(10^{13}\right)$ contained in a sapphire cell, a vapour pressure 100 times lower than expected from the saturated vapour pressure curve is observed [6]. Adding a small piece of Ta foil, the vapour pressure is further reduced by at least four orders of magnitude. This behaviour is described by the Frenkel formula

$$
\tau=\tau_{0} \exp \left(-E_{\mathrm{A}} / k T\right),
$$

which gives the adsorption time $\tau$ as a function of the adsorption energy $E_{\mathrm{A}}$, the temperature $T$, and $\tau_{0}$ which is of the order of $10^{-12} \mathrm{~s}$. Clearly, high temperatures and wall materials of small adsorption energies for the element in question are favourable.

In this respect, the inert elements such as the noble gases are best suited for containment in cells. However, the chemical inertness goes along with a large energy gap to the first excited atomic level, so that laser radiation in the ultraviolet spectral region is needed to excite the atom. Until recently, only pulsed lasers delivered sufficient power during the pulse to generate ultraviolet light by frequency doubling. The low-duty cycle of such lasers $\left(10^{-7}\right)$ requires a confinement of the low abundance atoms in a cell. Here the need of a pulsed laser coincides with the chemical inertness which makes the cell technique advantageous. It should be remarked, however, that a population of metastable states circumvents the problem of ultraviolet resonance lines, and allows the use of cw lasers (see the contribution to this volume by R. Neugart).

\section{Experimental setup}

Figure 1 shows the experimental setup used for laser spectroscopy on $\mathrm{Hg}$ isotopes at ISOLDE. A slightly modified version (allowing the temperature of the cell to be raised to about $400{ }^{\circ} \mathrm{C}$ ) has been used in the case of the $\mathrm{Cd}$ isotopes. The mass separated ions were collected for about two nuclear half-lives on a foil. Then, they were released as atoms into a quartz vessel by heating the foil and were excited from the ${ }^{1} \mathrm{~S}_{0}$ ground state to the first excited ${ }^{3} \mathrm{P}_{1}$ state by frequency-doubled laser light at $\lambda=253.7 \mathrm{~nm}$ or $326.1 \mathrm{~nm}$. The resonance light was observed by a photo multiplier. Frequency calibration was accomplished by simultaneously observing the resonance radiation of stable even-even isotopes in a Zeeman-shifted reference cell. For the investigation of $\mathrm{Au}$ isotopes, temperatures in the range of $1100-1500^{\circ} \mathrm{C}$ are needed. 


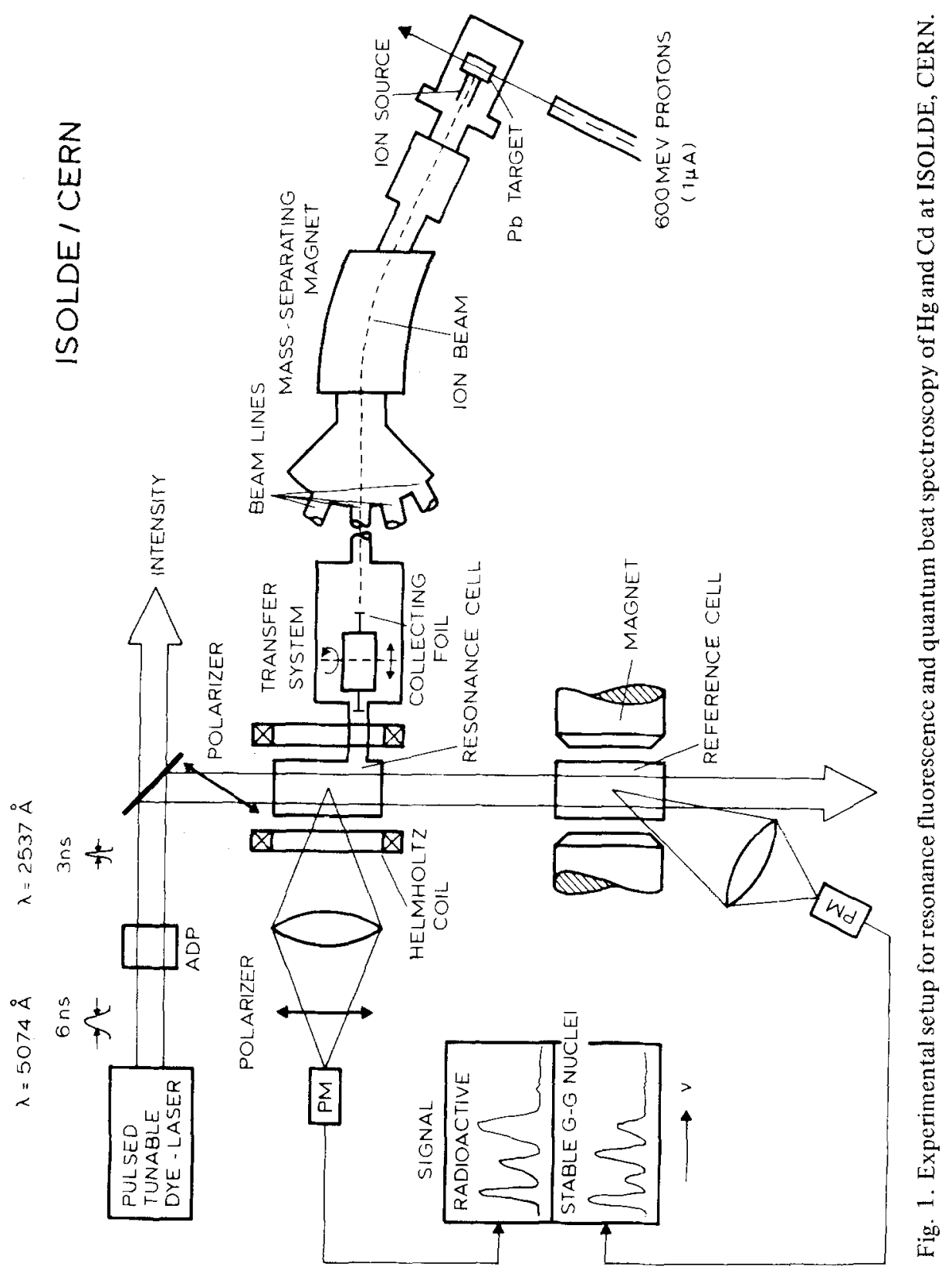


This was achieved by use of sapphire as cell material. The cell was located in an Mo tube (fig. 2) which was heated directly by a current of up to $700 \mathrm{~A}$.

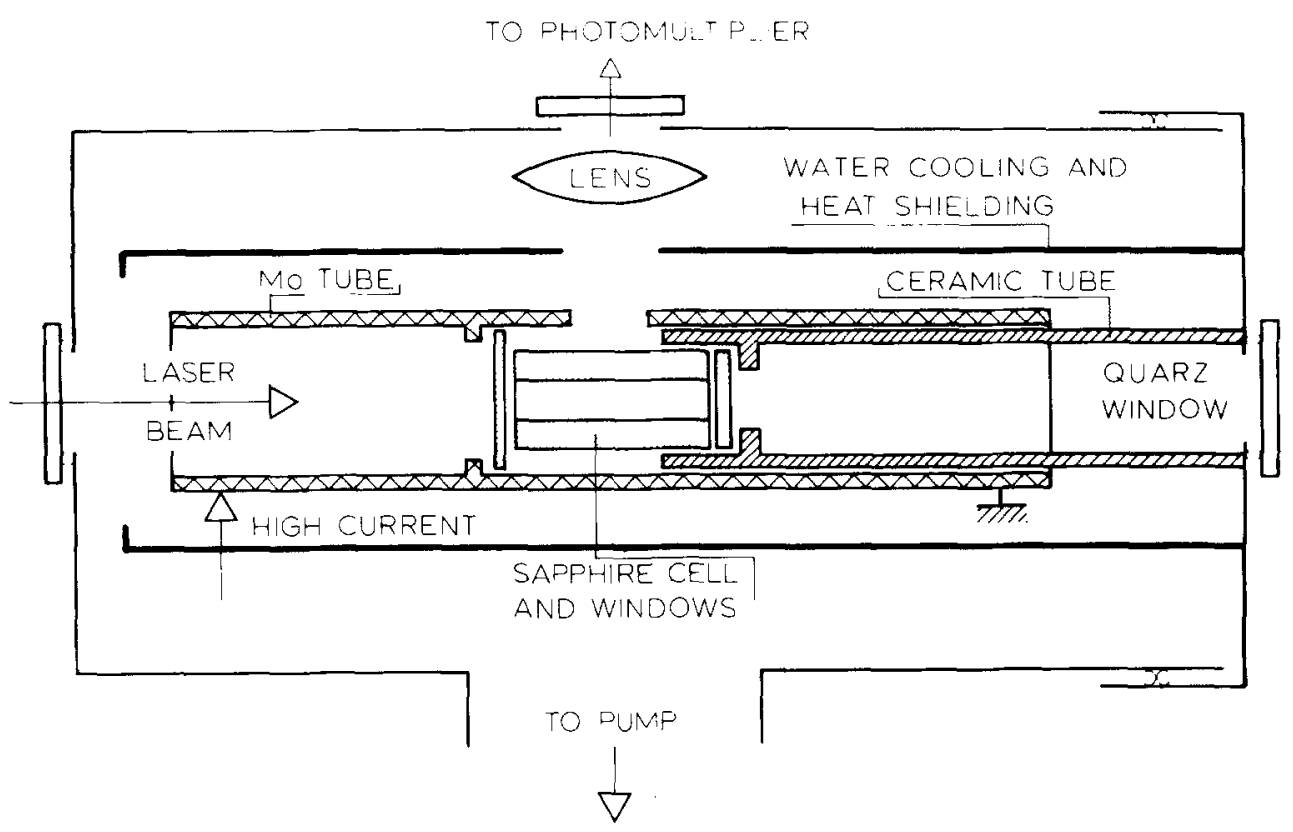

Fig. 2. Cell-oven system for the optical spectroscopy of $\mathrm{Au}$ at ISOLDE, CERN. Maximum temperature is $1500{ }^{\circ} \mathrm{C}$.

\section{Resonance fluorescence spectroscopy}

Figure 3 shows as an example the HFS and IS of the odd isotope ${ }^{185} \mathrm{Hg}$ for the ground and isomeric states, and fig. 4 the signals of ${ }^{197} \mathrm{Au}$ (stable) and ${ }^{193} \mathrm{Au},{ }^{192} \mathrm{Au}$, ${ }^{191} \mathrm{Au}$, and ${ }^{190} \mathrm{Au}$. Since the HFS and IS are large for these heavy elements, the Doppler broadening of the lines can be tolerated. However, this is no longer the case for $\mathrm{Cd}$, where the resolution suffers substantially by the large line broadening.

In these experiments, the measuring time was of the order of $10 \mathrm{~min}$. About $10^{9}$ atoms in the resonance cell were necessary for $\mathrm{Hg}, 10^{10}$ for $\mathrm{Cd}$ and $10^{12}$ for $\mathrm{Au}$. Clearly, improvements with respect to resolution and sensitivity are desirable. 


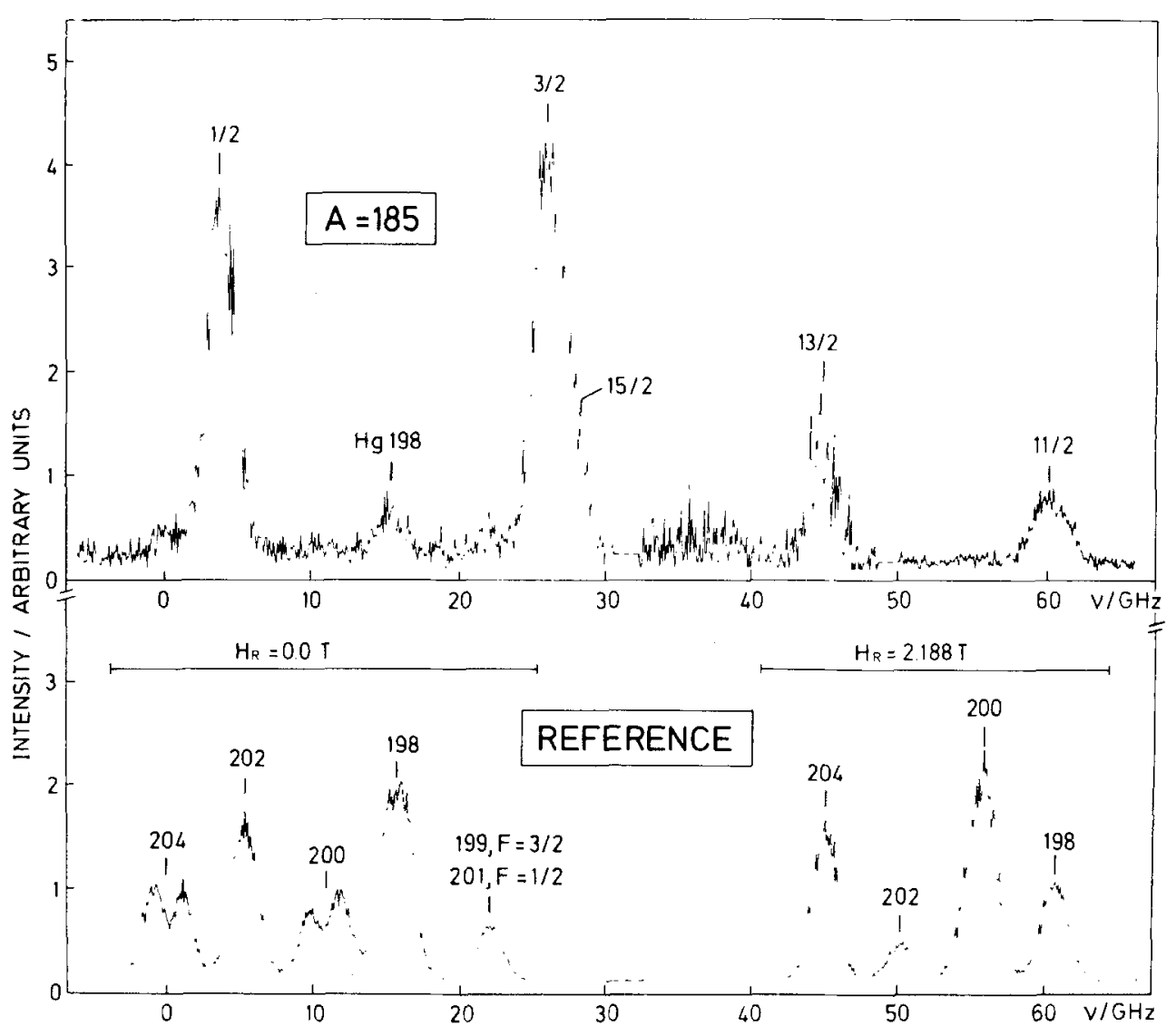

Fig. 3. Resonance fluorescence signal of ${ }^{185} \mathrm{Hg}(I=1 / 2)$ and ${ }^{185 \mathrm{~m}} \mathrm{Hg}$ $(I=13 / 2)$ in the ${ }^{1} \mathrm{~S}_{0}-{ }^{3} \mathrm{P}_{1}, \lambda=253.7 \mathrm{~nm}$ transition. The huge isomer shift is evident. In the lower part, the simultaneously measured resonance fluorescence of stable $\mathrm{Hg}$ isotopes is shown. A magnetic field was used to establish a frequency scale, using the known Zeeman splitting. 


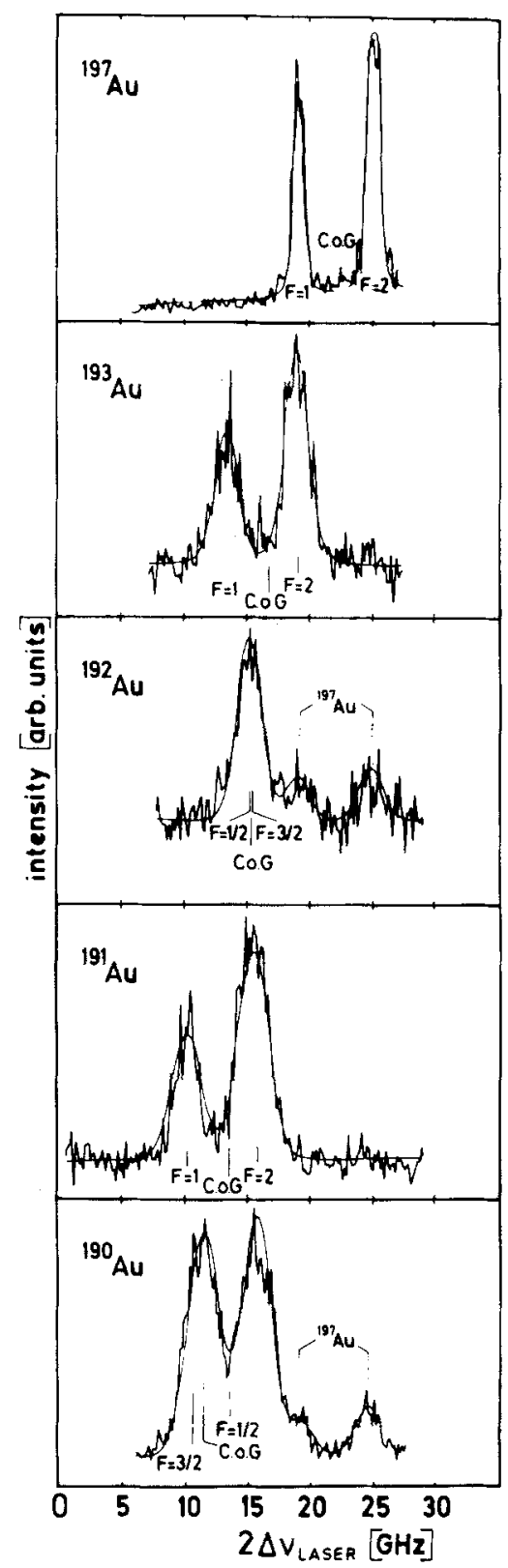

Fig. 4. Resonance fluorescence signal of Au isotopes recorded in a single laser scan. Top: signal of stable ${ }^{197} \mathrm{Au}$ in an atomic beam. Others: signal of neutron-deficient isotopes. In each case, about $10^{13}$ atoms were confined in the sapphire cell at temperatures above $1300^{\circ} \mathrm{C}$. The solid lines represent the result of a least-squares fit. The $F$ quantum number of the atomic ground state and the center of gravity are indicated. In the case of ${ }^{192} \mathrm{Au}$ and ${ }^{190} \mathrm{Au}$, stable $\mathrm{Au}$ was put into the resonance vessel in order to passivate the walls of the cell. 


\section{Towards higher resolution}

\subsection{QUANTUM BEAT SPECTROSCOPY}

Using a pulsed laser, it is straightforward to excite two closely spaced levels coherently and to observe the frequency difference of these levels as intensity modulation of the exponential atomic decay. The quantum beat technique [12] has been used to determine the nuclear spins of the $\mathrm{Hg}$ isotopes from a measurement of the Zeeman splitting in a weak magnetic field. The spin is determined via the $g_{F}$-factor

$$
g_{F}=g_{J}[F(F+1)-J(J+1)-I(I+1)] /[2 F(F+1)] .
$$

The maximum energy splitting which can be measured depends on the pulse length of the laser, the rise time of the single photon events, and the sampling technique used. Recently, a multiscaling unit with 2000 channels and a sampling rate of $500 \mathrm{MHz}$ has been developed at Mainz [13]. Although this sampling rate is extremely high, the maximum resolvable frequency is in general far below the HFS splitting. However, the splitting can be determined by exciting the $\left(\Delta m_{F}=2\right)$-levels near a level-crossing point [13]. The level-crossing point can be determined by variation of the magnetic field and measurement of the quantum beat frequency. From the magnetic field of the crossing, the zero-field HFS splitting is then calculated with an accuracy limited only by the natural line width $\Delta v_{\text {nat }}=1 / 2 \pi \tau$, with $\tau$ being the lifetime of the excited atomic state. In the case of the test experiment on ${ }^{199} \mathrm{Hg}$, an accuracy of $0.1 \mathrm{MHz}$ was achieved, which is comparable to the precision of a radio frequency experiment. In this experiment, two photo multipliers were placed at $90^{\circ}$ with respect to each other as well as to the direction of the magnetic field. Hence, the exponential decay can be eliminated and the pure modulation signal is isolated with an excellent signalto-noise ratio (fig. 5.)

\subsection{POLARIZATION SPECTROSCOPY}

Another way to circumvent the Doppler broadening is the application of polarization spectroscopy [14] with two counter propagating beams. An atom with a velocity $v_{z}$ parallel to the laser beams sees in its rest frame one of the laser beams at a frequency $\nu^{+}=v_{0}\left(1+v_{z} / c\right)$, the other at $\nu^{-}=v_{0}\left(1-v_{z} / c\right)$. If the laser bandwidth is negligible, the atom can only interact with both beams at the resonance frequency $\nu_{0}$ and a velocity $v_{z}=0$, thus eliminating the Doppler broadening. The strong circularlypolarized beam saturates the $\Delta m=+1$ transitions in the case of right-handed circular polarization. As a consequence, the vapour loses its absorptive as well as its dispersive power for right-handed light and turns from an isotropic to an anisotropic medium. A 

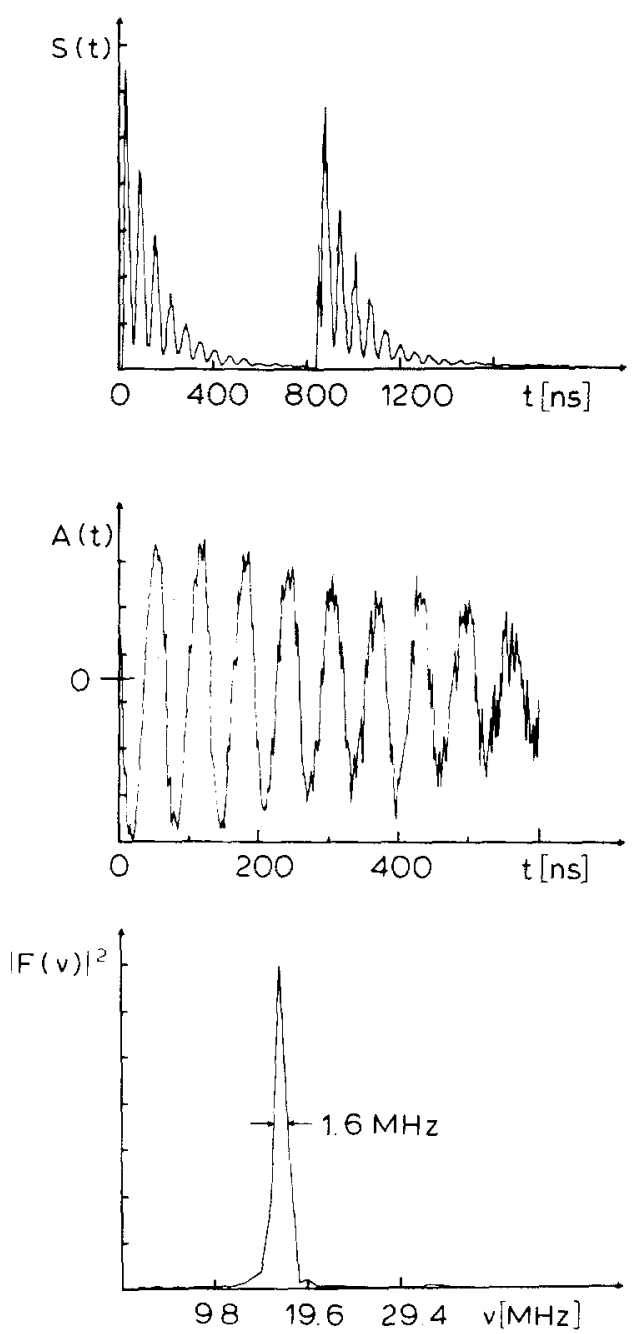

Fig. 5. Quantum beat signal obtained for ${ }^{204} \mathrm{Hg}$ in a weak magnetic field in the ${ }^{1} \mathrm{~S}_{0^{-}}{ }^{3} \mathrm{P}_{1}, \lambda=253.7 \mathrm{~nm}$ transition. The simultaneously recorded signals of two photo multipliers (top) are subtracted and normalized by their sum (middle). By Fourier transformation, a clean frequency spectrum is obtained (bottom).

linearly polarized beam probes this anisotropy in that its plane of polarization is tilted if it interacts with the same atom. This is observed through an analyzer perpendicular to the polarizer. The technique has been applied for a measurement of ${ }^{195} \mathrm{Au}$ [6]. However, the method suffers from the selection of a velocity group resulting in a reduced sensitivity in the case of a laser with narrow bandwidth and from the lack of cell materials without bire-fringence at high temperatures. 


\subsection{OTHER DOPPLER-FREE SCHEMES IN RESONANCE CELLS}

A technique similar to polarization spectroscopy is saturation spectroscopy [15] as used by the Berlin group for the investigation of noble gases [16]. In order to use $\mathrm{cw}$ dye lasers working in the visible spectral region, a metastable state is populated by a radio-frequency discharge and an atomic transition starting from this level is used for spectroscopy. Another scheme is two-photon spectroscopy, where the previously mentioned trick of counter propagating beams is used (see the contribution to this volume by B. Cagnac). By absorption of one photon from each beam at the rest frame frequencies $\nu^{+}$and $\nu^{-}$, respectively, the Doppler effect cancels again. The atom is excited via a virtual intermediate state to an excited state of the same parity as the ground state at energy $E=2 h v_{0}$.

Until now, this technique has only been used for the investigation of stable isotopes. The reason is the simultaneous need of high power and spectral purity of the laser. In the case of the two-photon spectroscopy of minute samples of radioactive atoms, a high excitation probability is demanded of a reasonable percentage of the atoms confined in the cell (diameter of the beam waist of the laser $\geqslant 1 \mathrm{~mm}$ ). As a consequence, only pulsed lasers with peak power of about $1 \mathrm{MW}$ in order to saturate the optical transition can be applied. The bandwidth of a pulsed dye laser system (typically of the order of $1 \mathrm{GHz}$ ) can be narrowed down to less than $100 \mathrm{MHz}$ by etalons of high finesse in the cavity of the dye laser or external filtering of the laser light $[17,18]$.

Besides being Doppler free, two-photon spectroscopy offers some attractive advantages for the investigation of short-lived isotopes: (i) all atoms interact with the laser light independent of their velocities, (ii) wall-scattered laser light can be blocked by filters since the wave length of excitation is different from that of the observation of the resonance, and (iii) in the case of $n \mathrm{~s}^{2}{ }^{1} \mathrm{~S}_{0}-n \mathrm{sn}$ 's ${ }^{1} \mathrm{~S}_{0}$ transitions (e.g. in the case of $\mathrm{Hg}$ ), the full transition strength is combined in one resonance line, immediately yielding the IS between different isotopes. Because of these advantages, we have tested this method in the case of $\mathrm{Hg}$ and $\mathrm{Au}$. Figure 6 shows an example of the $6 s^{2}{ }^{1} S_{0}-6 s 7 s^{1} S_{0}$ transition in a natural isotopic mixture of $\mathrm{Hg}$ isotopes [18]. For this experiment, the bandwidth of the laser is narrowed to $75 \mathrm{MHz}$ in the visible region by a high-finesse intracavity etalon and the cavity modes are synchronized with the etalon modes by a galvano plate [18]. Frequency doubling is needed to generate the UV laser light.

The poor signal-to-background ratio (fig. 6) is due to a tiny spectral impurity of the laser in the visible region which is boosted by the frequency-doubling process and by the two-photon adsorption, resulting in a sizeable background as explained in fig. 7. Since signals like the one displayed in fig. 6 are only obtained after time-consuming careful adjustments of the laser, the prospects of this technique are not promising for the on-line investigation of short-lived isotopes. 


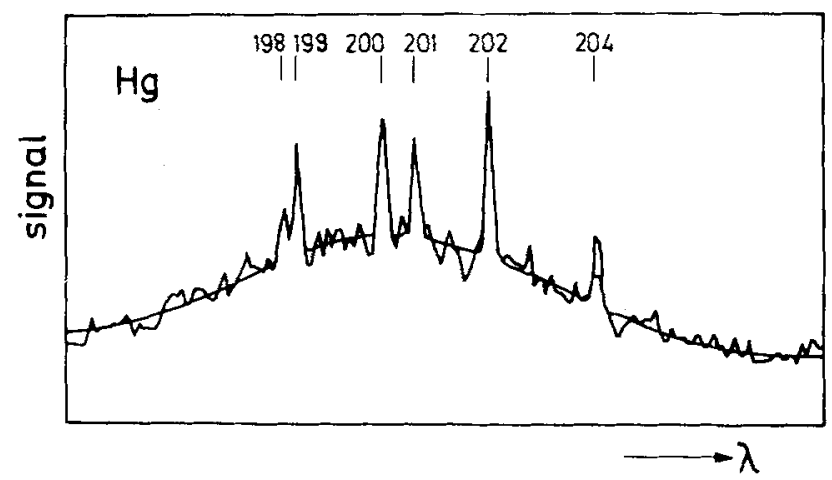

Fig. 6. Resonance fluorescence signal of a natural mixture of $\mathrm{Hg}$ observed in the $6 s 7 s^{1} \mathrm{~S}_{0}-6 s 6 \mathrm{p}^{3} \mathrm{P}_{1}, \lambda=408.1 \mathrm{~nm}$ transition after two-photon transition from the $6 \mathrm{~s}^{2}{ }^{1} \mathrm{~S}_{0}$ ground state to the $6 \mathrm{~s} 7 \mathrm{~s}^{1} \mathrm{~S}_{0}$ excited state at $\lambda=312.8 \mathrm{~nm}$. The smooth line represents a least-squares fit with 6 gaussian curves for the narrow resonances (FWHM $=300 \mathrm{MHz}$, relative height $=1$ ) and 6 gaussian curves for the broad background $(\mathrm{FWHM}=11 \mathrm{GHz}$, relative height $=0.24$, centered at the narrow resonances $)$.

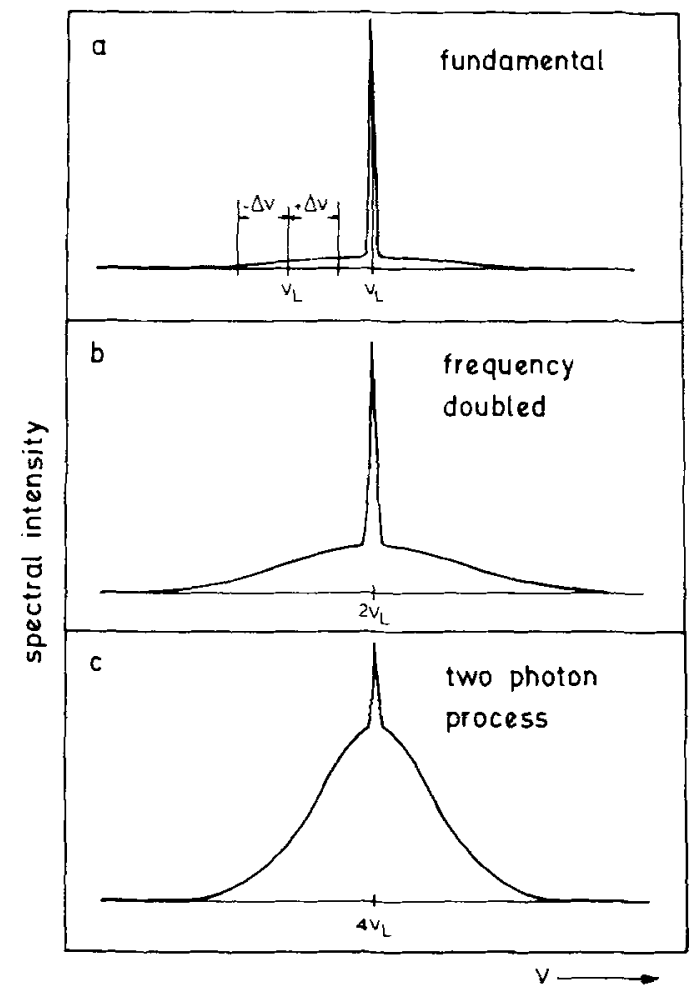

Fig. 7. Explanation of the background observed in the two-photon experiment on $\mathrm{Hg}$ (fig. 6). Assuming a profile of the laser intensity $I_{\mathrm{L}}(v)$ as shown in (a) (ratio of the laser intensity with narrow bandwid th to that of the broad background $=20$ in the center of the profile), the ratio decreases to 4 after passing the frequency. doubling crystal (b) where not only harmonic generation but also mixing takes place. Hence, the profile of the laser intensity in the UV is given by

$$
I(2 \nu)=\int_{-\infty}^{+\infty} I_{\mathrm{L}}(\nu-\Delta \nu) I_{\mathrm{L}}(\nu+\Delta \nu) \mathrm{d} \Delta \nu .
$$

The analogous process happens in the two-photon transition (c) decreasing further the ratio to 0.5 . The $\mathrm{Hg}$ signals of fig. 6 are explained by an intensity ratio of $60: 1$ in the spectrum of the visible laser light. 
Finally, it should be mentioned that the RADOP technique (sect. 6.1) enables high-precision measurements of ground-state HFS. In this case the resolution is only limited by the relaxation time of the nuclear orientation achieved by optical pumping.

\section{Towards higher sensitivity}

Since the number of radioactive atoms is generally small and limited, it is very important that the fraction of the atoms interacting with the laser beam be as high as possible. Therefore, small volume resonance cells are demanded. On the other hand, the smaller the resonance vessel, the more laser light is scattered from the walls into the photo detector. Hence a compromise has to be found. In the case of the experiments on $\mathrm{Hg}, \mathrm{Cd}$ and $\mathrm{Au}$, this led to a percentage of free atoms in the cell interacting with the laser beam of the order of $1 \%$. Still the wall-scattered laser light was the principal factor in limiting the sensitivity. This problem can, in principle, be solved by exciting higher lying atomic levels and observation of the fluorescence light at a different wave length or - as discussed above - by using two-photon excitation. In addition to wall adsorption, other factors will then become important in limiting sensitivity:

- the small solid angle of the photon detector,

- the low quantum efficiency of the photo cathode,

- the broad-band fluorescence of the wall material,

- the sensitivity of the photon detector to the radioactivity.

A very effective way out of these limitations is the application of the different kinds of RADOP techniques or other schemes of non-optical detection.

\subsection{NUCLEAR RADIATION DETECTED OPTICAL PUMPING (RADOP)}

The $\beta$-RADOP technique was first tried out by Besch et al. [19]. The aim of these experiments was the determination of the spins and the magnetic moments of short-lived alkali atoms. The nuclei were produced directly in the resonance vessel by $(\mathrm{p}, \mathrm{n}),(\mathrm{d}, \mathrm{n})$ and $(\alpha, \mathrm{p})$ reactions. In these experiments, noble gases were used for several purposes: They served, (i) as target material for the production of the investigated isotope, (ii) as stopping material for the produced isotope having a considerable momentum, and (iii) as buffer gas for OP. Two methods were applied to orient the nuclei: Direct OP of the unstable atom or spin exchange by collisions of the radioactive atom with stable $\mathrm{Rb}$ polarized by $\mathrm{OP}$. More details can be found in ref. $[20]$.

The first measurements of the IS by the RADOP technique were obtained with the investigation of the odd neutron-deficient ground state $\mathrm{Hg}$ isotopes at ISOLDE. In these experiments, a tunable light source was accomplished by a spectral 
lamp containing a stable even $\mathrm{Hg}$ isotope and placed in the pole gap of a magnet. In this way, the HFS and IS in the optical transition could be scanned. Although in $\beta$ RADOP only one event per nucleus can be observed, the high efficiency of the $\beta$ detector and the low background enabled measurements with only $10^{3}-10^{4}$ atoms present in the resonance vessel $\left({ }^{181} \mathrm{Hg}, T_{1 / 2}=3.6 \mathrm{~s}\right)$.

Another means of detecting nuclear orientation is $\gamma$-RADOP $[21,22]$. Here the OP light is linearly polarized in order to obtain nuclear alignment. Maximum anisotropy signals are obtained by calculating the normalized difference spectrum between the $\gamma$ spectra obtained with light linearly polarized parallel and perpendicular to the direction of the magnetic field. With this technique, the HFS and IS of the isomeric state of ${ }^{199} \mathrm{Hg}$ was determined [22]. In addition, the method can be exploited to establish or check nuclear level schemes. Today, powerful lasers can be applied in order to achieve a high degree of nuclear orientation (see the contributions to this volume by D. Murnick and by $\mathrm{C}$. Bemis). The tunability and monochromaticity of these light sources can be used to select the most appropriate transition for OP. The high $\mathrm{cw}$ power available allows one to reach pumping times in the $\mu \mathrm{s}$ regime. Thus it becomes possible to apply laser spectroscopy to nuclei with half-lives below $1 \mathrm{~ms}$, provided that within the nuclear lifetime the electron shell of these atoms can be restored after the production process. The first measurement of a nucleus with a half-life of $1 \mathrm{~ms}$ was reported by Bemis et al. on ${ }^{240 \mathrm{~m}} \mathrm{Am}$ by fission-fragment RADOP. Here, the fission fragments provide the signature of the shape isomer, and the change in the angular distribution of the fission fragments is the monitor of the optical resonance.

Generally, it can be concluded that the shorter the nuclear lifetime, the more superior is the observation of nuclear decay to the observation of the optical photons.

\subsection{OTHER SCHEMES}

A promising alternative to resonance fluorescence and RADOP is the resonance ionization spectroscopy (RIS) [23-25], where the atom is stepwise excited to (i) a Rydberg level where it is field ionized, or (ii) photo-ionized by an excitation into the continuum, or (iii) photo-ionized by a transition to an auto-ionizing state. Very recently, a first application of this technique has been reported for the investigation of unstable Eu isotopes [26].

At Mainz, we tried to enhance the selectivity of this technique by combination of the RIS scheme with a measurement of the mass of the ion by the time-offlight method and to increase the sensitivity of the method by generating a pulsed atomic beam in order to beat the low-duty cycle of a high peak power pulsed laser system. Figure 8 shows the setup. The radioactive atoms are adsorbed on a surface. Pulsed desorption from the backing is achieved by heating it with the beam of a first pulsed laser. After an appropriate delay, the desorbed atoms are stepwise excited and ionized 


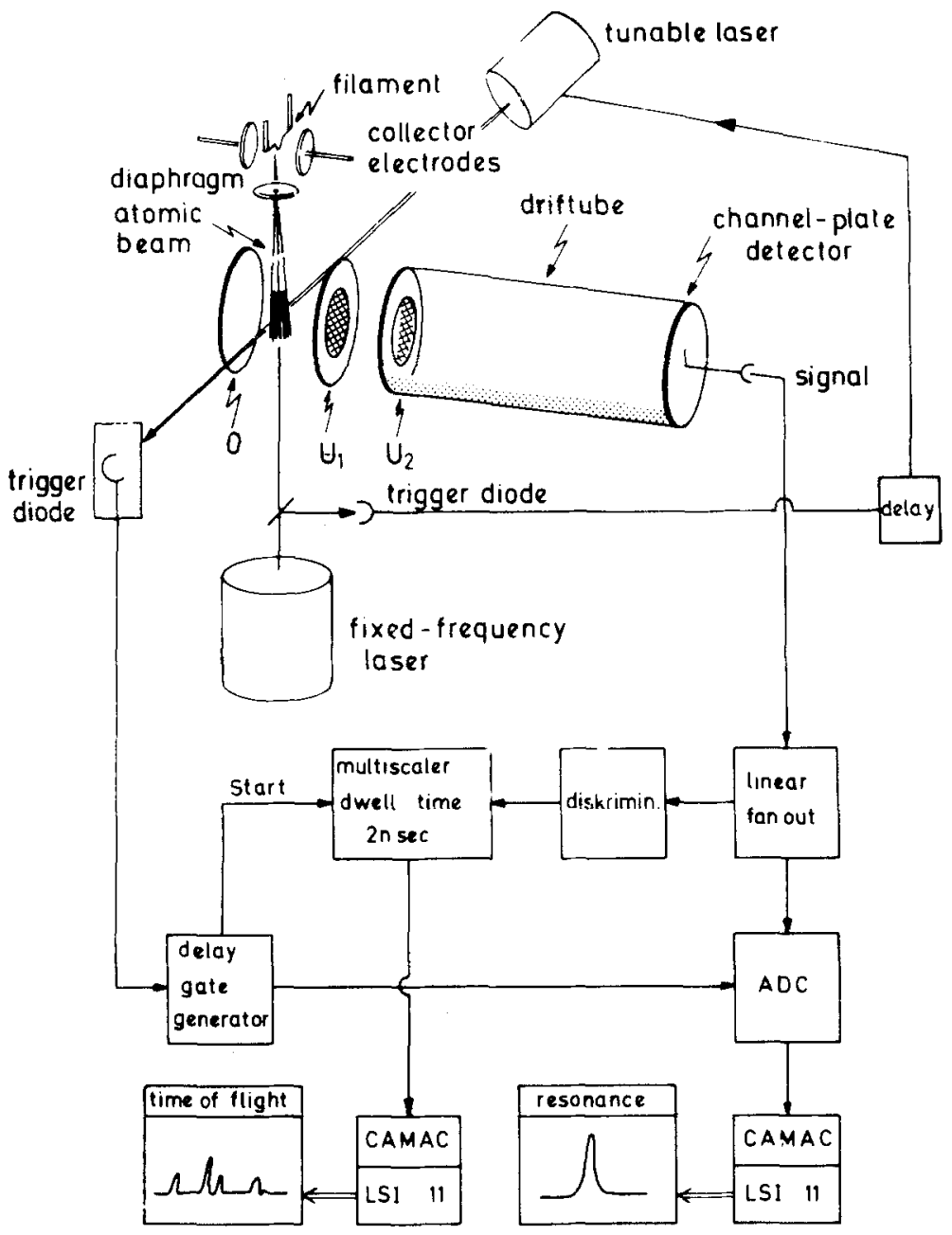

Fig. 8. Experimental setup for resonance ionization spectroscopy in combination with a measurement of the mass of the ion by time of flight. A $\mathrm{cw}$ thermal atomic beam is formed by heating the filament. Alternatively, a pulsed atomic beam can be obtained by laser desorption with the help of another pulsed laser with fixed frequency.

by a second laser. The ions created in resonance are accelerated in an electric field and allowed to drift to a channel plate where they are detected. Since the short laser pulse (pulse length $=5 \mathrm{~ns}$ ) represents an ideal start signal, and a time focus at the detector can be obtained with appropriate voltages in the region of acceleration, the mass resolution is in excess of 1000 .

Until now, the combination of laser desorption, resonance ionization spectroscopy, and time of flight has been applied by us only to the case of In. The results are encouraging, but no final conclusions can be drawn on the applicability of this method for a study of short-lived nuclei. 
Resonance ionization spectroscopy with mass selectivity by time of flight has been tested by us in the cases of $\mathrm{Xe}, \mathrm{Au}, \mathrm{Hg}, \mathrm{U}$ and $\mathrm{Pu}$. Figure 9 gives a sketch of the transitions used in these experiments. In cases where two-photon transitions

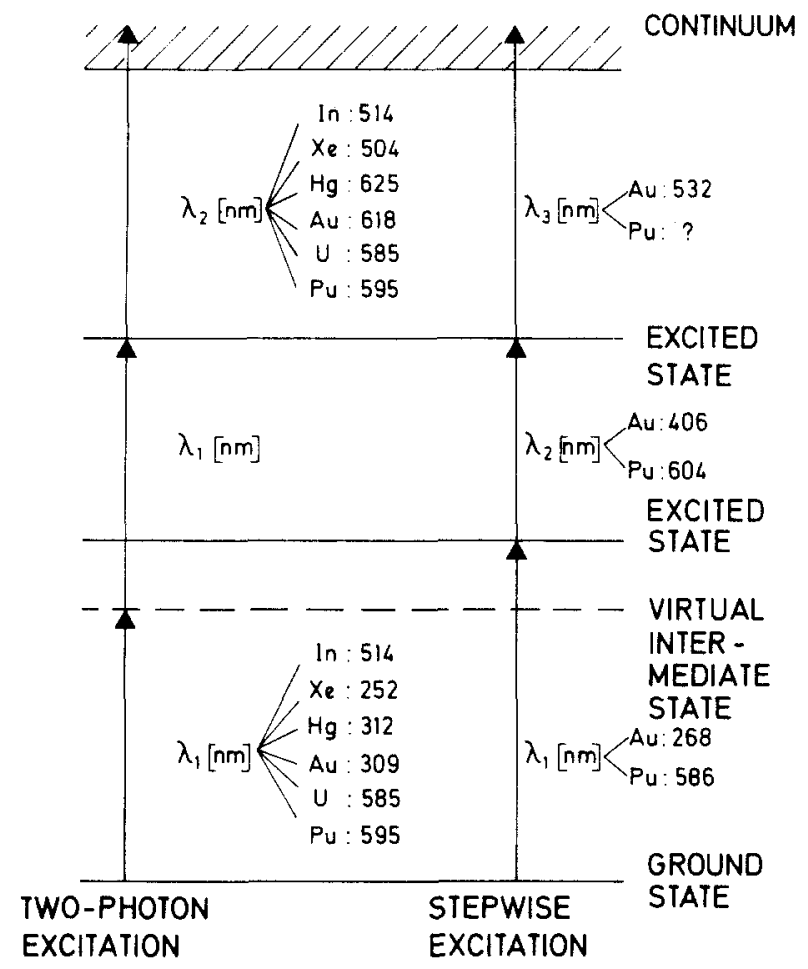

Fig. 9. Resonance ionization schemes used for the investigation of $\mathrm{In}, \mathrm{Xe}, \mathrm{Hg}, \mathrm{Au}, \mathrm{U}$ and $\mathrm{Pu}$. Broken lines indicate virtual intermediate state used in the twophoton experiments, solid lines represent real states. The wave length for the different experiments are indicated.

are needed in the ultraviolet spectral region, the rest gas ionized by the strong laser beam represents a strong source of background (fig. 10). The non-resonant ionization of the rest gas plays no role when visible laser light or stepwise excitation are used. As an example, figs. 11 and 12 show the result of a trace analysis experiment of $\mathrm{Pu}$ [9] . In this case, a total number of $10^{13} \mathrm{Pu}$ atoms are electrolytically deposited on a Re filament and evaporated by heating the foil to temperatures up to $1800{ }^{\circ} \mathrm{C}$. The time-of-flight spectrum (fig. 11) shows the RIS signal of Pu and the non-resonant photo ionization of some $\mathrm{Pu}$ compounds. In addition, spurious signals of ions show up which are released from the foil during the period of heating up the filament, but 

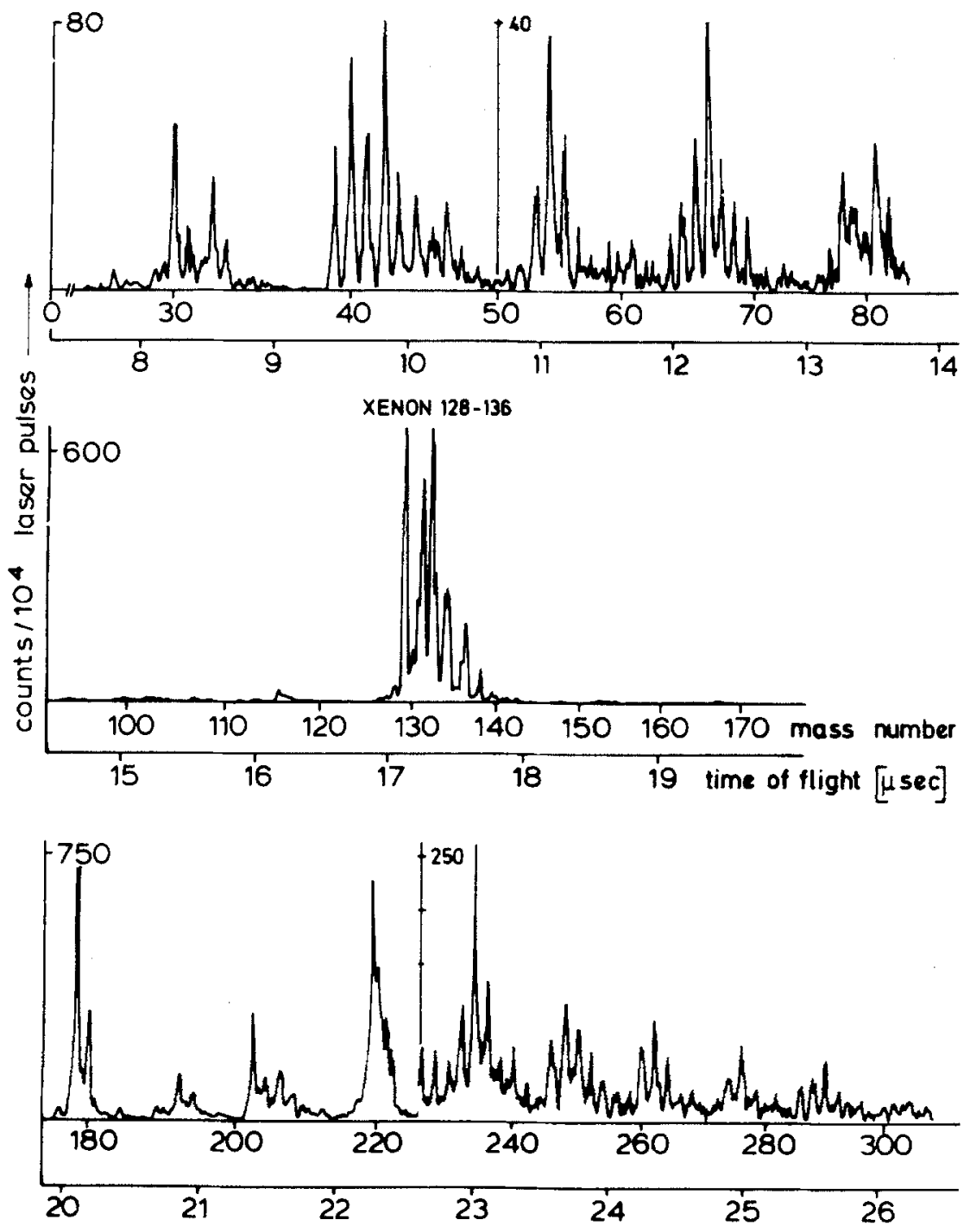

Fig. 10. Time-of-flight spectrum obtained by resonance ionization spectroscopy on $\mathrm{Xe}$ (partial pressure $=5 \times 10^{-6} \mathrm{mbar}$ ). The non-resonant photo-ionized residual gas (partial pressure $=2 \times 10^{-7} \mathrm{mbar}$ ) is observed over the whole mass range recorded. The laser parameters are: pulse energy $=5 \mu \mathrm{J}$, pulse energy $=3 \mathrm{~ns}, \lambda=252.4 \mathrm{~nm}$, Bandwidth $=0.02 \mathrm{~nm}$, repetition rate $=10 \mathrm{~Hz}$.

are not suppressed by means of the collector electrodes (fig. 8). Figure 12 shows a scan of the two-photon resonance of Pu obtained by gating on the Pu mass and scanning the frequency of the laser.

The sensitivity of this experiment is limited by the low repetition rate of the laser of $10 \mathrm{~Hz}$ and the small solid angle $\left(10^{-4}\right.$ of $\left.4 \pi\right)$ due to the distance of $40 \mathrm{~mm}$ 


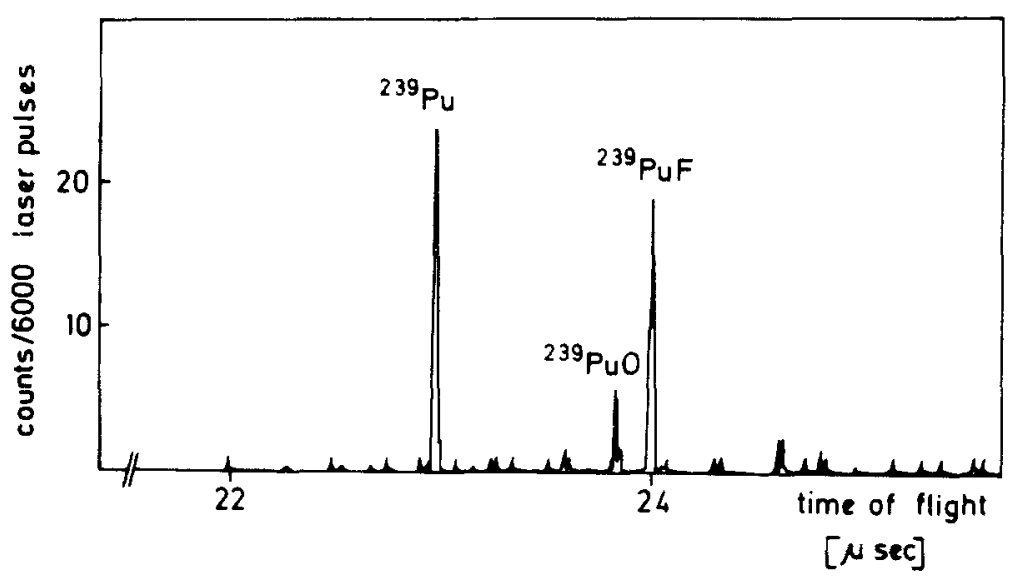

Fig. 11. Time-of-flight spectrum of Pu obtained by resonance ionization spectroscopy. A total amount of $6 \mathrm{ng}$ of ${ }^{239} \mathrm{Pu}$ was used for this experiment.

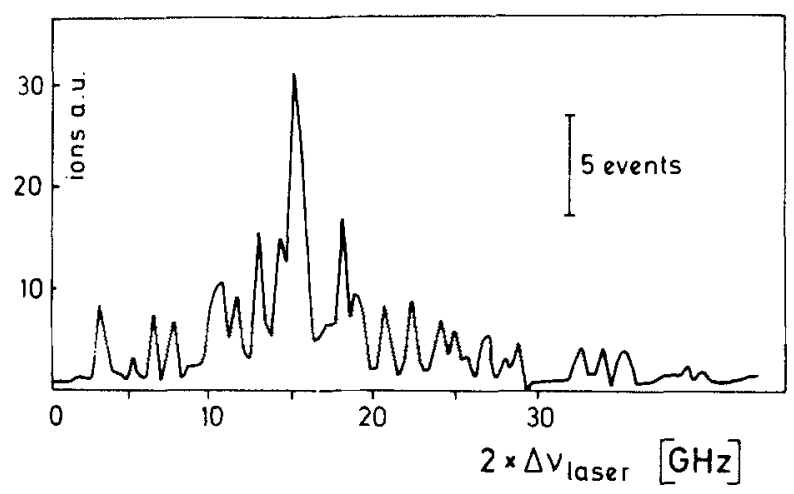

Fig. 12. Resonance ionization signal of ${ }^{239} \mathrm{Pu}$ versus the frequency of the laser. The laser parameters are: pulse energy $=3.5 \mathrm{~mJ}$, power density $=30 \mathrm{MW} / \mathrm{cm}^{2}$, pulse length $=10 \mathrm{~ns}, \lambda=595 \mathrm{~nm}$, bandwidth $=0.76 \mathrm{~Hz}$, repetition rate $=10 \mathrm{~Hz}$.

between the foil and the laser beam ( $1 \mathrm{~mm}$ diameter). It can possibly be enhanced by a factor of $10^{6}$ by the use of a pulsed beam (see above) or a high repetition $\mathrm{Cu}$ vapour laser in combination with stepwise excitation and ionization. Figure 13 demonstrates the considerable improvement of the signal-to-background ratio achievable by RIS in comparison to the detection of the resonant photons of the first step of excitation. In this case, an oven containing macroscopic amounts of $\mathrm{Au}$ is used. At an oven temperature of about $1200{ }^{\circ} \mathrm{C}$, the RIS signal is completely saturated. At $T \simeq 1100^{\circ} \mathrm{C}$, 


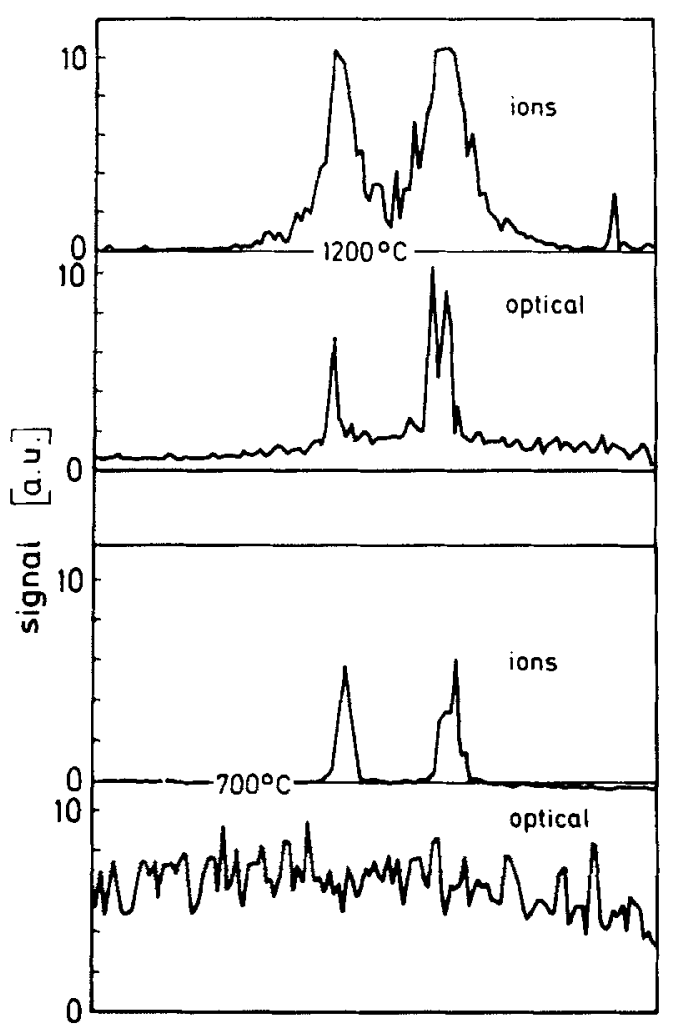

Fig. 13. Comparison of the stepwise resonance ionization spectroscopy with the detection of resonance fluorescence in the ${ }^{2} \mathrm{~S}_{1 / 2}{ }^{2} \mathrm{P}_{1 / 2}, \lambda=242.9 \mathrm{~nm}$ transition of $\mathrm{Au}$. The spectra are taken simultaneously. The temperatures of the oven for the production of the thermal beam of $\mathrm{Au}$ are indicated.

the resonance light disappears in the noise of the promptly scattered laser light. Even at $700{ }^{\circ} \mathrm{C}$, where the vapour pressure is decreased by 5 orders of magnitude in comparison with the density at $1200^{\circ} \mathrm{C}$, an excellent ion signal is recorded.

\section{Some results}

The application of atomic spectroscopy in resonance cells to the $\mathrm{Hg}$ isotopic chain turned out to be very fruitful. Spins, magnetic and electric moments, and isotope shifts could be determined for a long isotopic series. The changes of the charge radii in the light isotopes represent perhaps the most exciting result of these systematic investigations. The bistability of the nuclear shape of the $\mathrm{Hg}$ nuclei with a nearly magic proton number but a neutron number half way between closed shells, 
has been discussed at former conferences and in recent reviews [27]. Therefore, I would like to concentrate on some general aspects of the transitional nuclei in the $\mathrm{Hg}$ region.

Isotope shifts and nuclear deformation: Figure 14 shows a plot of the available $\delta\left\langle r^{2}\right\rangle^{A}, A^{\prime}$ data of the $\mathrm{Pb}$ to Os isotopes. In order to calculate $\delta\left\langle r^{2}\right\rangle$ by means of

$$
\delta\left\langle r^{2}\right\rangle_{\mathrm{f}}^{A, A^{\prime}}=c_{1}^{A, A^{\prime}} \cdot \lambda^{A, A^{\prime}}=c_{1}^{A, A^{\prime}}\left(\delta\left\langle r^{2}\right\rangle^{A, A^{\prime}}+\left(c_{2}^{A, A^{\prime}} / c_{1}\right) \delta\left\langle r^{4}\right\rangle^{A, A^{\prime}}+\ldots\right),
$$

the ratios $c_{k} / c_{1}$ are taken from a calculation by Seltzer [28] and $c_{1}^{A, A^{\prime}}$ is determined by use of electronic or muonic X-ray data, the Droplet model, or by the relativistic Dirac-Fock calculations [29]. With the exception of the nuclear shape transitions observed for the $\mathrm{Hg}$ isotopes below $N=106$, the nuclear charge radii shrink quite regularly with decreasing neutron number. A small kink of the average slopes around $N=118$ indicates a subshell closure or an onset of steadily increasing or decreasing deformation at this neutron number. A pronounced odd-even staggering is superimposed over and above the general shrinking of the charge radii, and the magnitude of the staggering effect is surprisingly independent of the proton number. Despite the uncertainties of the individual elements $c_{1}$ (estimated to less than 10\%), almost parallel lines obtained for the charge radii of different elements are interpreted as being due to identical changes in nuclear shape. This can be seen by the simple twoparameter model

$$
\delta\left\langle r_{\mathrm{f}}^{2}\right\rangle_{\mathrm{f}}^{A, A^{\prime}}=\delta\left\langle r^{2}\right\rangle_{\text {volume }}^{A, A^{\prime}}+\delta\left\langle r^{2}\right\rangle_{\text {shape }}^{A, A^{\prime}}
$$

where the first part accounts for the change of the nuclear charge radius of the hypothetically spherical nucleus. The second part is due to a change in nuclear shape. The volume contribution is known to be well described by the Droplet model in the case of the heavy nuclei [31].

Since $\delta\left\langle r^{2}\right\rangle$ Droplet changes only by $1 \%$ between $\mathrm{Pb}$ and Os for isotope pairs with the same neutron numbers, the shape-dependent part of (4) is responsible for different average slopes of the individual isotopic chains. The prediction of the spherical Droplet model is plotted in fig. 14 for the case of Au. It is obvious that the Au isotopes shrink less with decreasing neutron number than expected by a pure volume effect described by the Droplet model. In the framework of the two-parameter model (4), an increase of $\delta\left\langle r^{2}\right\rangle$ shape is observed which is due to increasing static or dynamic nuclear deformation or increasing diffuseness of the nuclear surface. In heavier nuclei, the dominant contribution to the change of $\delta\left\langle r^{2}\right\rangle_{\text {shape }}$ stems from a change in static or dynamic quadrupole deformation $\left\langle\beta^{2}\right\rangle$. Considering only these effects, the second term of (4) reduces to 


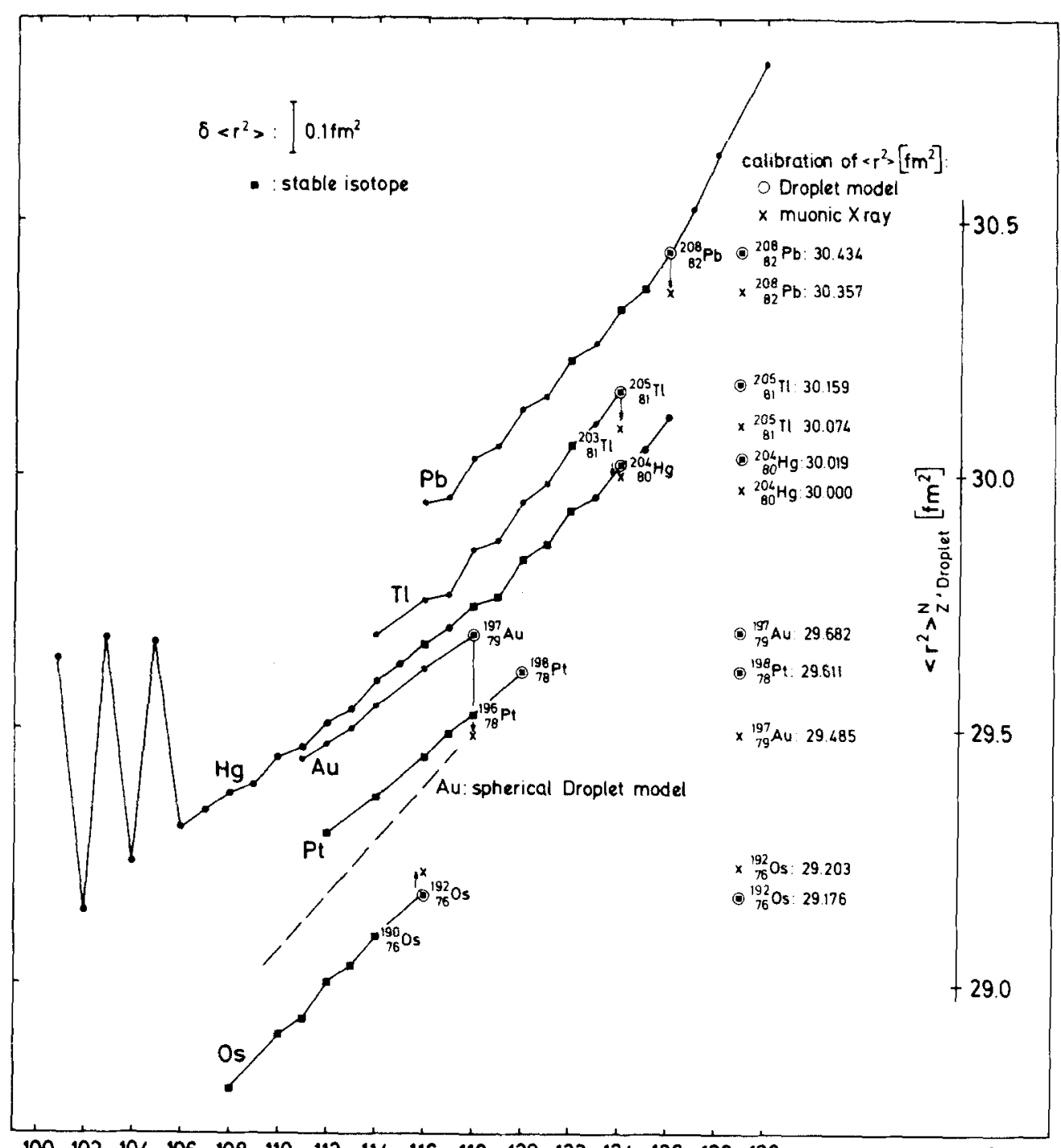

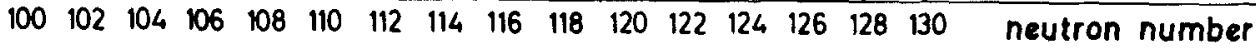

Fig. 14. Plot of the charge radii available in the $\mathrm{Pb}$ to Os region. Squares with a circle indicate the $\left\langle r^{2}\right\rangle$ value obtained by the Droplet model with theoretically calculated deformation [31]. Using muonic X-ray data for calibration, the data of the isotopic chains have to be shifted as indicated by the arrows and crosses. 


$$
\delta\left\langle r^{2}\right\rangle_{\text {shape }}^{A, A^{\prime}} \approx \delta\left\langle r^{2}\right\rangle_{\beta}^{A, A^{\prime}}=\frac{3}{4 \pi} R_{0}^{2} \delta\left\langle\beta^{2}\right\rangle^{A, A^{\prime}}
$$

with $R_{0}=1.2 A^{1 / 3} \mathrm{fm}$ and the quadrupole parameter $\beta$. If $\delta\left\langle r^{2}\right\rangle_{\text {volume }}$ is identified with $\delta\left\langle r^{2}\right\rangle_{\text {Droplet }}$ calculated with the help of the spherical Droplet model [30], this leads to

$$
\delta\left\langle r^{2}\right\rangle_{\mathrm{f}}^{A, A^{\prime}} \cong \delta\left\langle r^{2}\right\rangle_{\text {Droplet }}^{A, A^{\prime}}+\frac{3}{4 \pi} R_{0}^{2} \delta\left\langle\beta^{2}\right\rangle^{A, A^{\prime}} .
$$

and allows a determination of the change of the quadrupole deformation along an isotopic chain relative to a reference isotope. The deformation parameters of the $\mathrm{Hg}$ and $\mathrm{Au}$ isotopes are plotted in fig. 15. The trends of the deformation parameters

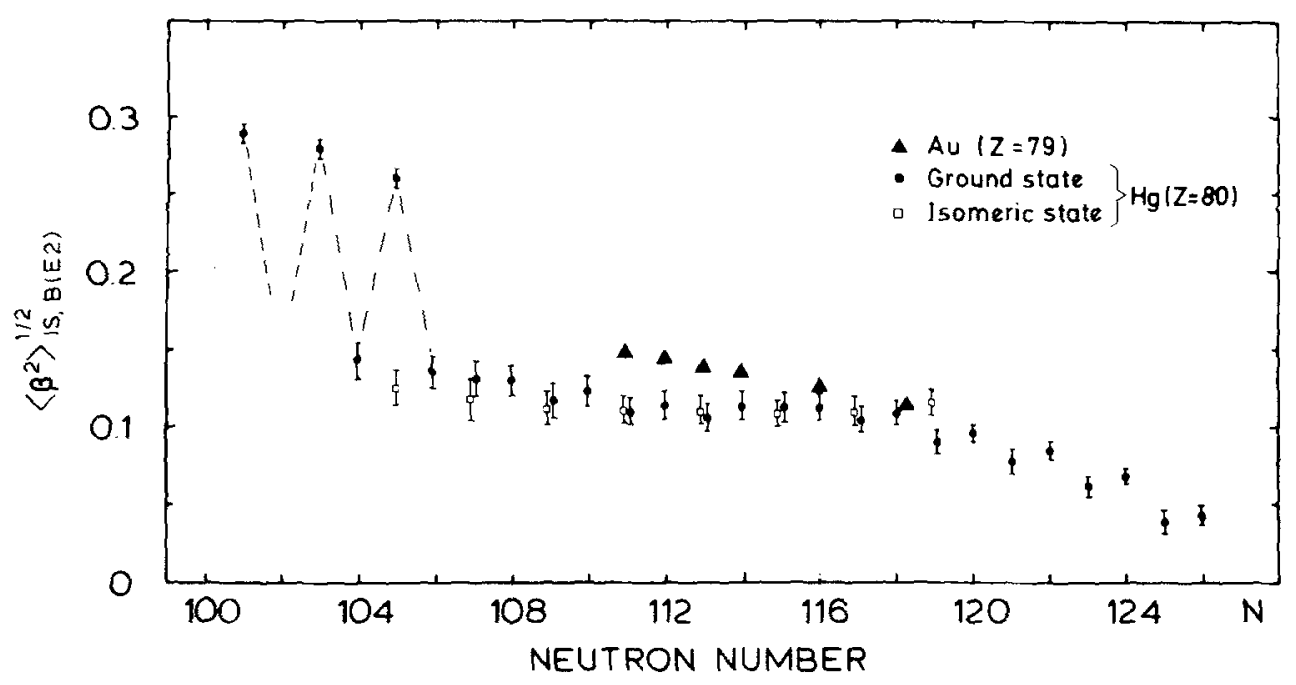

Fig. 15. Quadrupole deformation parameters of $\mathrm{Hg}$ and $\mathrm{Au}$. The deformation parameters of $\mathrm{Hg}$ are obtained by a combined analysis of IS and $B(E 2)$ data. The deformation of the $\mathrm{Au}$ isotopes are evaluated from the IS assuming $\beta=0.113$ for ${ }^{197} \mathrm{Au}$.

are consistent with the expectation that the deformation increases with the distance from the magic numbers $Z=82$ and $N=126$. Whereas the $\mathrm{Hg}$ data show an instability of the nuclear shape as discussed briefly above, no drastic effect is observed for Au down to ${ }^{190} \mathrm{Au}$, the lightest $\mathrm{Au}$ isotope optically investigated. The data available do not allow any conclusions to be drawn as to whether the strong deformation expected around $N \simeq 105$ is reached in a smooth way or by a sudden onset of deformation caused by the nearby magic proton number. 
Odd-even staggering: The gross features of the variation of the charge radii as a function of neutron number are quite well understood [31]. However, finer details such as the odd-even staggering of the IS are far from being satisfactorily interpreted by theory. As an example, the odd-even staggering of the $\mathrm{Hg}$ isomers is shown in fig. 16 , calculated according to

$$
\gamma^{A+1}=2 \delta\left\langle r^{2}\right\rangle^{A, A+1} / \delta\left\langle r^{2}\right\rangle^{A, A+2} ; A \text { even }
$$

The regular trend is remarkable. Several qualitative explanations of this effect have been tried, but no theory explains the experimental observations unambiguously and quantitatively. It can be hoped, however, that the information obtained by the systematic investigation of long isotopic chains will help to solve the puzzle, as was done in the case of spectroscopic quadrupole moments (see below).

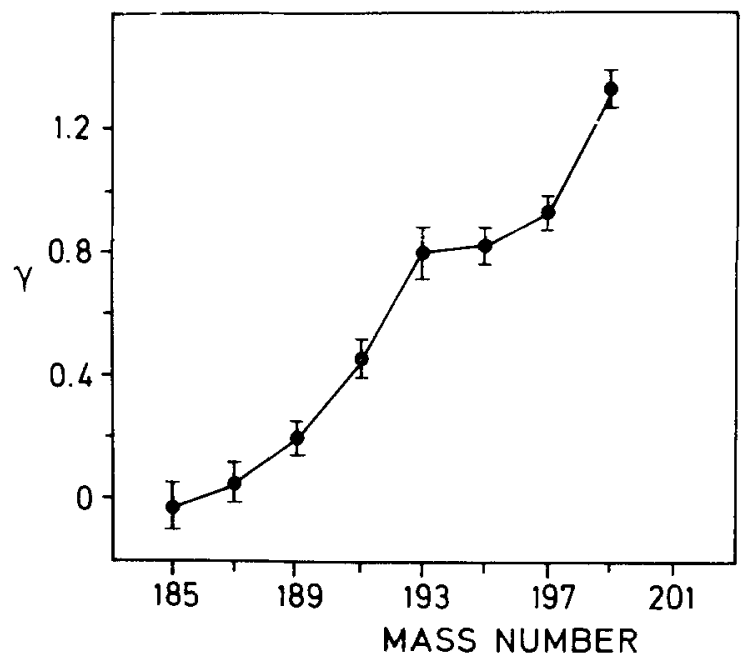

Fig. 16. Odd-even staggering parameter of the $I=13 / 2$ isomers of $\mathrm{Hg}$.

In addition, reliable $\left\langle r^{2}\right\rangle$ values are needed to fix the relative optical $\delta\left\langle r^{2}\right\rangle$ data in an absolute scale. This would allow one to study the odd-even staggering of isotone shifts and to observe the shell closure effects at $Z=82$ along chains of constant neutron number.

Quadrupole moments: The spectroscopic quadrupole moments of transitional nuclei have been an unsolved puzzle for a long time. Neither size nor sign could be interpreted. The measurement of the quadrupole moments of the eight isomeric states ${ }^{199 m} \mathrm{Hg}-{ }^{185 m} \mathrm{Hg}$ gave the first hint that the occupation number of a subshell 
and the type of coupling might be as important as the intrinsic deformation. The regular trend of the spectroscopic quadrupole moments, the purity of the $i_{13 / 2}$ neutron states, and the observation of decoupled bands in the heavy isomers led to the assumption of a transition from Coriolis decoupling to strong coupling as a function of decreasing neutron number [3]. Ragnarsson [32] explained the quadrupole moments quantitatively (fig. 17) by keeping the intrinsic deformation constant and by calcula-

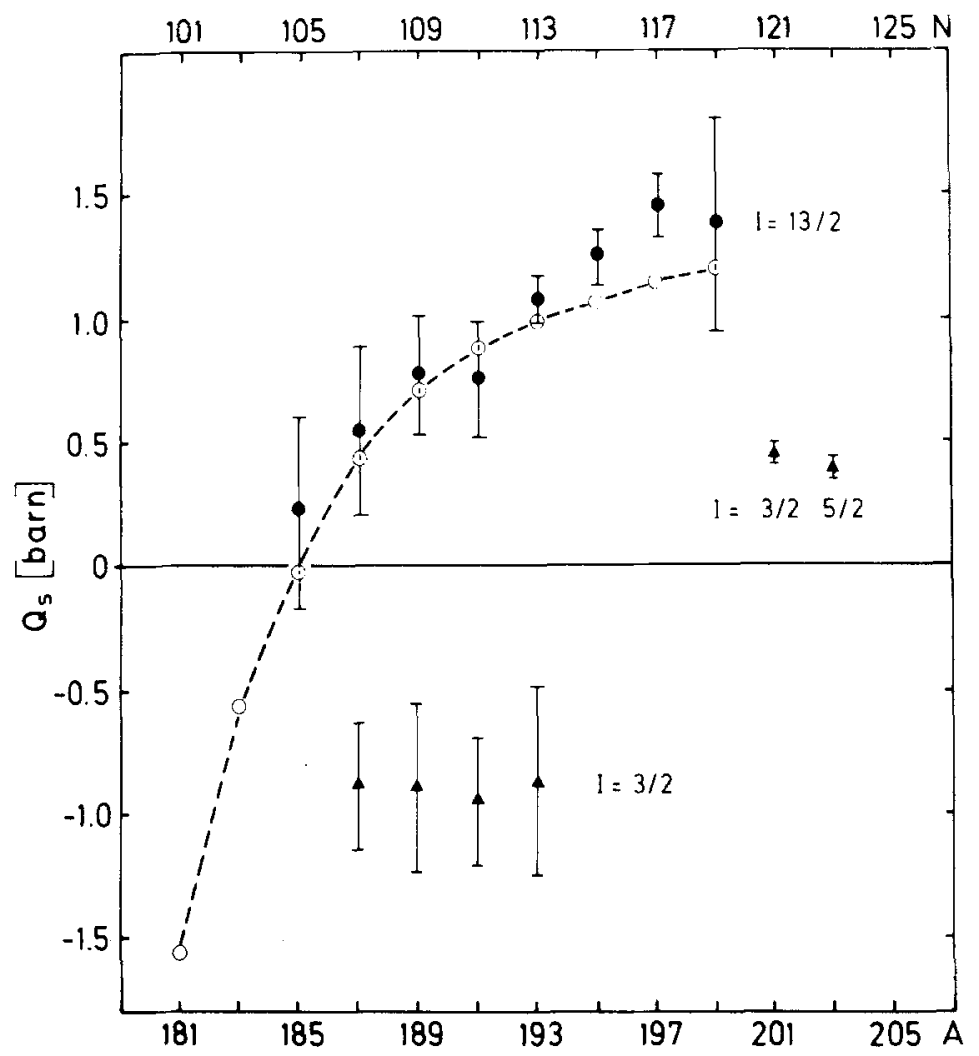

Fig. 17. Quadrupole moments of $\mathrm{Hg}$ isotopes. The full dots represent the experimental values. The broken line gives the theoretical quadrupole moments calculated by Ragnarsson for the isomers [32]. In these calculations, the deformation parameter was kept constant at $\beta=-0.12$.

ting the amplitudes of the $\Omega$ projection $\mathrm{d}_{\alpha \Omega}^{j}$ within the particle-plus-rotor model as a function of the occupation of the $i_{13 / 2}$ subshell. Then the projection formula reads

$$
Q_{\mathrm{s}}=Q_{0} \quad 3 \sum_{\Omega}\left[\left(\mathrm{d}_{\alpha \Omega}^{j} \Omega\right)^{2}-I(I+1)\right] /[(I+1)(2 I+3)]
$$

with $Q_{0}$ being the intrinsic quadrupole moment. 
Following these lines, Ekström was able to interpret a large number of spectroscopic quadrupole moments of transitional nuclei, as reported at the Helsingør Conference [33]. No information exists on the quadrupole moments of the $\mathrm{Au}$ isotopes with the exception of the only stable nucleus ${ }^{197} \mathrm{Au}$. These moments are especially interesting because of the existence of long-lived high-spin isomeric states built up by unpaired $h_{11 / 2}$ protons and/or $i_{13 / 2}$ neutrons.

\section{Conclusions}

Optical spectroscopy of radioactive isotopes in resonance cells has been proved to be a powerful technique for the determination of nuclear properties in long isotopic chains. Especially the polarization by optical pumping and the observation of the anisotropy of the nuclear radiation represents an extremely sensitive monitor for the optical resonance. However, in each case the advantages and drawbacks of the cell technique have to be considered carefully.

\section{References}

[1] G. Huber et al., Z. Phys. A276(1976)187.

[2] J. Bonn et al., Phys. Lett. 38B(1972)308; Z. Phys. A276(1976)203.

[3] T. Kühl et al., Phys. Rev. Lett. 39(1977)180; Phys. Lett. 82B(1979)199; Phys. Rev. Lett. 43(1979)1376; and to be published.

[4] F. Buchinger et al., Hyp. Int. 9(1981)165; and to be published.

[5] H.L. Ravn, Phys. Rep. 54(1979)201.

[6] H.-J. Kluge et al., Z. Phys. A309(1983)187.

[7] J. Streib et al., Z. Phys. A (1985), in print.

[8] H.-J. Klugre et al., Rev. Sci. Instr. 55(1984)873.

[9] U. Krönert et al., Appl. Phys. B (1985), in print.

[10] H.-J. Kluge, in: Lasers in Nuclear Physics, Proc. of the Conference on Lasers in Nuclear Physics, Oak Ridge, April 1982, ed. C.E. Bemis and H.K. Carter, Nuclear Science Research Conference Series, Vol. 3 (Harwood Academic Publishers, Chur-London-New York, 1982) p. 137.

[11] H. Fischer et al., Z. Phys. A284(1978)3.

[12] S. Haroche, in: Topics in Applied Physics, Vol. 14 (Springer, Berlin-Heidelberg - New York, 1976).

[13] T. Blaich et al., Rev. Sci. Instr. 54(1983)706.

[14] C. Wiemann and T. Hänsch, Phys. Rev. Lett. 36(1976)1170.

[15] See, for example, W. Demtröder, in: Laser Spectroscopy (Springer, Berlin - HeidelbergNew York, 1981).

[16] H. Gerhard et al., Z. Phys. A292(1979)7; Appl. Phys. 22(1980)261; Hyp. Int. 9(1981)175.

[17] R. Wallenstein et al., Opt. Conm. 14(1975)353.

[18] H. Schaaf et al., to be published.

[19] H.J. Besch et al., Phys. Lett. 25B(1967)120.

[20] H.-J. Kluge, in: Progress in Atomic Spectroscopy, ed. W. Hanle and H. Kleinpoppen (Plenum, New York, 1979) Part B, p. 727. 
[21] U. Cappeller and W. Mazurkewitz, J. Magn. Res. 10(1973)15.

[22] J. Bonn et al., Z. Phys. A272(1975)375.

[23] R.V. Ambartzumian et al., JETP Lett. 13(1971)217.

[24] V.S. Letokhov, in: Nonlinear Laser Chemistry, Springer Series in Chemical Physics, Vol. 22 (Springer, Berlin-Heidelberg-New York, 1983).

[25] G.S. Hurst et al., Rev. Mod. Phys. 51(1979)767.

[26] G.D. Alkhazov et al., JETP Lett. 37(1983)274.

[27] See, for example, P.G. Hansen, Ann. Rev. Nucl. Part. Sci. 29(1979)69; or P. Jacquinot and R. Klapisch, Rep. Prog. Phys. 42(1979)773; or E.W. Otten, Nucl. Phys. A354(1981)471c.

[28] E.C. Seltzer, Phys. Rev. 188(1969)1916.

[29] A. Rosen et a1., Z. Phys. A316(1984)157.

[30] W.D. Myers et al, Nucl. Phys. A336(1980)267.

[31] W.D. Myers et al., Nucl Phys. A410(1983)61.

[32] I. Ragnarsson, in: Future Directions in Studies of Nuclei far from Stability, Nashville 1979, ed. J.H. Hamilton et al. (North-Holland, Amsterdam, 1980) p. 367.

[33] C. Ekström, in: 4th Int. Conf. on Nuclei far from Stability. Helsingør 1981, ed. P.G. Hansen and O.B. Nielsen, CERN Report No. 81-09, p. 12.

\section{Discussion}

H.A. Schuessler: Could you compare the resolution of the new ISOLDE which you gave as 5000 (and 30000 with reduced intensity) with the old ISOLDE?.

H.J. Kluge: $\quad$ The existing ISOLDE II facility has a resolution of about 500 . Thus, the planned ISOLDE III provides an improvement of one to two orders of magnitude.

R. Coussement: Is the mass separation power of ISOLDE III large enough to perform nuclear mass spectroscopy?

H.J. Kluge: $\quad$ Even a resolution of 30000 would not be sufficient since for $A=100$, it corresponds to $\Delta M \approx 3 \mathrm{MeV}$. The high resolution aims mainly for obtaining an isotopically clean beam by rejecting isobaric masses. Direct mass determinations of nuclei far from stability, however, are planned by the Orsay and Mainz groups, using two different techniques. The expected accuracy is of the order of $10^{-6}$ to $10^{-7}$.

R. Coussement: Does ISOLDE III plan to add an electrostatic analyzer?

H.-J. Kluge: $\quad$ No.

$R$. Coussement: Can the emittance of the ion source be better?

H.J. Kluge: The emittance of the source is already quite good. On has to choose a compromise between good emittance and sufficient pumping speed for the outgassing of the target. For ISOLDE III, the possible use of slit ion sources is planned. 